\title{
On a Generalized $2+1$ Dispersive Water Wave Hierarchy
}

\author{
By \\ Pilar R. GordoA, Nalini Joshi** and Andrew PICKeRING*
}

\begin{abstract}
We present a generalized non-isospectral dispersive water wave hierarchy in $2+1$ dimensions. We characterize our entire hierarchy and its underlying linear problem using a single equation together with its corresponding non-isospectral scattering problem. This then allows a straightforward construction of linear problems for the entire generalized $2+1$ hierarchy. Reductions of this hierarchy then yield new integrable hierarchies in $1+1$ dimensions, and also new integrable hierarchies of ordinary differential equations, all together with their underlying linear problems. In particular, we obtain a generalized $P_{I V}-P_{I I}$ hierarchy; this includes as special cases both a hierarchy of ODEs having the fourth Painlevé equation as first member, and also a hierarchy of ODEs having the second Painlevé equation as first member. All of these hierarchies of ordinary differential equations, as well as their underlying linear problems, are new; both the $P_{I V}$ hierarchy and the $P_{I I}$ hierarchy obtained here are different from those which have previously been given.
\end{abstract}

\section{$\S 1$. Introduction}

Out of the huge variety of different kinds of completely integrable systems that are known today, one particular class has in recent years been attracting increasing interest, namely those associated to non-isospectral scattering problems [1]-[17]. There are many reasons for this interest; one is the connection that exists between non-isospectral scattering problems and linear problems for ODEs [9]; another is the fact that very little appears to be understood

Communicated by T. Kawai, December 27, 2000.

2000 Mathematics Subject Classification(s): Primary 35Q58; Secondary 34M55.

* Area de Fisica Teórica, Facultad de Ciencias, Edificio de Fisica, Universidad de Salamanca, 37008 Salamanca, Spain.

** Department of Pure Mathematics, University of Adelaide, Adelaide 5005, Australia. 
about the actual process of Inverse Scattering for such systems [1]-[3]; a third reason is, as observed in [13], [14], that it is possible to characterize an entire hierarchy and its underlying linear problem using a single associated partial differential equation (PDE) in $2+1$ dimensions together with its underlying non-isospectral scattering problem. This last idea was used in [13], [14] to construct new hierarchies of PDEs in $2+1$ dimensions, together with their non-isospectral scattering problems, along with new hierarchies of PDEs in $1+1$ dimensions having non-isospectral or isospectral scattering problems, and also new hierarchies of ordinary differential equations (ODEs) together with their underlying linear problems.

In the present paper we use the methodology developed in [13], [14] in order to construct a new non-isospectral variant of the dispersive water wave (DWW) hierarchy. Details of the standard $1+1$-dimensional hierarchy, in various different coordinates, can be found in [18]-[25]. Here we follow the choice of coordinates adopted in [24]. Reductions of our non-isospectral DWW hierarchy to ODEs then give new hierarchies of integrable systems of ODEs, together with their underlying linear problems. In particular we note that we obtain a generalized $P_{I V}-P_{I I}$ hierarchy; this includes as special cases both a hierarchy of ODEs having the fourth Painlevé equation $\left(P_{I V}\right)$ as first member, and also a hierarchy of ODEs having the second Painlevé equation $\left(P_{I I}\right)$ as first member. All of these results are new; in particular, we note that the $P_{I V}$ hierarchy and the $P_{I I}$ hierarchy obtained here are different from those which have previously been given, in [14] and [26], [27] respectively.

The layout of the paper is as follows. In Section 2 we construct our $2+$ 1-dimensional DWW hierarchy, together with its corresponding hierarchy of scalar and matrix linear problems, and also discuss reductions to hierarchies in $1+1$ dimensions. In Section 3 we consider reductions in components, and in Section 4 reductions to systems of ODEs; it is in Section 4 that we present our generalized $P_{I V}-P_{I I}$ hierarchy, along with our $P_{I V}$ and $P_{I I}$ hierarchies which appear as special cases, all together with their underlying scalar and matrix linear problems. In Section 5 we consider as explicit examples the first two members of these hierarchies. Section 6 is devoted to a summary and conclusions.

\section{§2. A Non-Isospectral 2+1 DWW Hierarchy}

Following the approach introduced in [13], [14], we begin by considering the $(2+1)$-dimensional system

$$
\mathbf{u}_{t}=\mathcal{R} \mathbf{u}_{\tau}+\mathbf{G}
$$


where $\mathbf{u}=(u, v)^{T}, \mathcal{R}$ is the recursion operator of the DWW hierarchy, $\mathbf{G}=$ $(g, 0)^{T}$, and $g$ is an arbitrary function of $t$ and $\tau$. This function $g$ is introduced into the system (1) by the non-isospectral condition, that is, by the equation satisfied by the spectral parameter in the corresponding Lax pair. The recursion operator of the DWW hierarchy is

$$
\mathcal{R}=\frac{1}{2}\left(\begin{array}{cc}
\partial_{x} u \partial_{x}^{-1}-\partial_{x} & 2 \\
2 v+v_{x} \partial_{x}^{-1} & u+\partial_{x}
\end{array}\right) .
$$

This can be written as the quotient of two Hamiltonian operators $B_{1}$ and $B_{2}$,

$$
\mathcal{R}=B_{2} B_{1}^{-1}
$$

where $B_{1}$ and $B_{2}$ are given by

$$
\begin{aligned}
& B_{2}=\frac{1}{2}\left(\begin{array}{cc}
2 \partial_{x} & \partial_{x} u-\partial_{x}^{2} \\
u \partial_{x}+\partial_{x}^{2} & v \partial_{x}+\partial_{x} v
\end{array}\right) \\
& B_{1}=\left(\begin{array}{cc}
0 & \partial_{x} \\
\partial_{x} & 0
\end{array}\right) .
\end{aligned}
$$

We note that in fact the DWW hierarchy is tri-Hamiltonian. However, we do not make use of the third Hamiltonian structure in this paper.

The system (1) has the Lax pair

$$
\begin{aligned}
\psi_{x x} & =\left[\left(\lambda-\frac{1}{2} u\right)^{2}+\frac{1}{2} u_{x}-v\right] \psi, \\
\psi_{t} & =\lambda \psi_{\tau}+\frac{1}{2}\left(\partial_{x}^{-1} u_{\tau}\right) \psi_{x}-\frac{1}{4} u_{\tau} \psi,
\end{aligned}
$$

where the spectral parameter $\lambda=\lambda(\tau, t)$ satisfies the condition

$$
\lambda_{t}=\lambda \lambda_{\tau}+\frac{1}{2} g
$$

We note that the system (1) is equivalent to an extension of the classical Boussinesq system having a non-isospectral scattering problem first given in [11], together with its Lax pair and Darboux transformation. In the case $g=0$ it is also equivalent to the $2+1$-dimensional extension of the nonlinear Schrödinger equation given in [2].

We now use the system (1) and the corresponding non-isospectral scattering problem (6)-(7) to generate our $(2+1)$-dimensional DWW hierarchy and at the same time the corresponding hierarchy of non-isospectral scattering problems. The idea [13], [14] is that, for suitably specified flow times $t$ and $\tau$, the 
system (1) can be understood as representing a generic member of the hierarchy and (6)-(7) its underlying scattering problem. We then use these equations to iterate between succesive flows and also their linear problems; note that we also iterate on the function $\mathbf{G}$. We take as base equation for this proccess of iteration the system

$$
\mathbf{u}_{t_{1}}=\mathcal{R} \mathbf{u}_{y}+\mathcal{R}^{2} \mathbf{G}_{0}+\mathcal{R} \mathbf{G}_{1}+\mathbf{G}_{2}
$$

When performing the iteration each $\mathbf{G}_{i}=\left(g_{i}, 0\right)^{T}$ and $\lambda$ are considered to be functions of all possible flow times $t_{j}$ and $y$, but not of $x$. The system (9) can be written locally by setting $u=w_{x}$;

$$
\begin{aligned}
(10) w_{x t_{1}}= & \frac{1}{2}\left[\left(w_{x} w_{y}-w_{x y}\right)_{x}+2 v_{y}\right] \\
& +\frac{1}{4} g_{0}\left(\left(x w_{x}^{2}-x w_{x x}-w_{x}\right)_{x}+4 v+2 x v_{x}\right)+\frac{1}{2} g_{1}\left(x w_{x}\right)_{x}+g_{2}, \\
\text { (11) } v_{t_{1}}= & \frac{1}{2}\left[v_{x} w_{y}+2 v w_{x y}+w_{x} v_{y}+v_{x y}\right] \\
& +\frac{1}{4} g_{0}\left(2 x w_{x x} v+2 x w_{x} v_{x}+4 w_{x} v+x v_{x x}+3 v_{x}\right)+\frac{1}{2} g_{1}\left(2 v+x v_{x}\right) .
\end{aligned}
$$

This system has the linear problem

$$
\begin{aligned}
\psi_{x x}= & {\left[\left(\lambda-\frac{1}{2} w_{x}\right)^{2}+\frac{1}{2} w_{x x}-v\right] \psi } \\
\psi_{t_{1}}= & \lambda \psi_{y}+\frac{1}{2}\left(\lambda g_{0} x+w_{y}+\frac{1}{2} g_{0} x w_{x}+g_{1} x\right) \psi_{x} \\
& -\frac{1}{4}\left(\lambda g_{0}+w_{x y}+\frac{1}{2} g_{0}\left(x w_{x}\right)_{x}+g_{1}\right) \psi,
\end{aligned}
$$

where the spectral parameter $\lambda$ satisfies the non-isospectral condition

$$
\lambda_{t_{1}}=\lambda \lambda_{y}+\frac{1}{2} \lambda^{2} g_{0}+\frac{1}{2} \lambda g_{1}+\frac{1}{2} g_{2}
$$

In order to iterate between successive flows of our hierarchy, and their corresponding scattering problems, we begin by writing a generic member of our $(2+1)$-dimensional hierarchy as

$$
\mathbf{u}_{t_{n}}=\mathbf{M}_{n}=\left(M_{n}, N_{n}\right)^{T},
$$

and the corresponding generic evolution equations for the eigenfunction $\psi$ and the spectral parameter $\lambda$ as

$$
\begin{aligned}
\psi_{t_{n}} & =\Gamma_{n} \psi_{y}+\frac{1}{2} P_{n} \psi_{x}-\frac{1}{4} P_{n, x} \psi, \\
\lambda_{t_{n}} & =\Lambda_{n} .
\end{aligned}
$$


We then obtain from (1), (7) and (8) the recursion relations

$$
\begin{aligned}
\mathbf{M}_{n} & =\mathcal{R} \mathbf{M}_{n-1}+\mathbf{G}_{n+1} \\
\Gamma_{n} & =\lambda \Gamma_{n-1} \\
\Lambda_{n} & =\lambda \Lambda_{n-1}+\frac{1}{2} g_{n+1} \\
P_{n} & =\lambda P_{n-1}+\partial_{x}^{-1} M_{n-1}
\end{aligned}
$$

where $\mathbf{M}_{n}=\left(M_{n}, N_{n}\right)^{T}$ and $\mathbf{G}_{n}=\left(g_{n}, 0\right)^{T}$. These recursion relations, together with the base equation (9) and its scattering problem and corresponding constraint on $\lambda$, then yield the hierarchy

$$
\mathbf{u}_{t_{n}}=\mathbf{M}_{n}=\mathcal{R}^{n} \mathbf{u}_{y}+\sum_{i=0}^{n+1} \mathcal{R}^{n+1-i} \mathbf{G}_{i},
$$

and the corresponding hierarchy of spectral problems

$$
\begin{aligned}
& \psi_{x x}=\left[\left(\lambda-\frac{1}{2} u\right)^{2}+\frac{1}{2} u_{x}-v\right] \psi, \\
& \psi_{t_{n}}=\lambda^{n} \psi_{y}+\frac{1}{2} P_{n} \psi_{x}-\frac{1}{4} P_{n, x} \psi,
\end{aligned}
$$

where $P_{n}$ is given by

$$
P_{n}=\partial_{x}^{-1} \sum_{i=-1}^{n-1} \lambda^{n-1-i} M_{i},
$$

and the spectral parameter $\lambda$ satisfies

$$
\lambda_{t_{n}}=\lambda^{n} \lambda_{y}+\frac{1}{2} \sum_{i=0}^{n+1} \lambda^{n+1-i} g_{i} .
$$

Here we have set $\mathbf{M}_{0}=\left(u_{y}+(1 / 2) g_{0}(x u)_{x}+g_{1}, v_{y}+(1 / 2) g_{0}\left(2 v+x v_{x}\right)\right)^{T}$ and $\mathbf{M}_{-1}=\left(g_{0}, 0\right)^{T}$. Note that here we have used the notation $\mathbf{M}_{-1}$ for convenience only; this is not meant to denote the inverse flow.

The hierarchy (22) is our generalized $2+1$-dimensional DWW hierarchy; this hierarchy is not to be found in the literature, although subcases have been discussed in [28]. We note that the second term on the right-hand side of (22) represents a non-isospectral deformation which gives rise to non-autonomous terms; we note also that for $n>1$ these are in the general case non-local. Linear combinations of the above flows can be obtained by allowing $\tau$ in (1) to be a vector. This then makes it trivial to add to (22) flows in $1+1$ dimensions (see Sections 4 and 5 later). 
Given a scalar linear problem, it is straightforward, as noted in [13], [14], to write down a corresponding matrix linear problem. Thus, corresponding to the scalar linear problem (23), (24), we have the matrix non-isospectral scattering problem

$$
\begin{aligned}
\boldsymbol{\Psi}_{x} & =F \boldsymbol{\Psi}, \\
\boldsymbol{\Psi}_{t_{n}} & =\lambda^{n} \boldsymbol{\Psi}_{y}+G \boldsymbol{\Psi},
\end{aligned}
$$

where $\boldsymbol{\Psi}=\left(\psi_{1}, \psi_{2}\right)^{T}$, the matrices $\mathcal{F}$ and $\mathcal{G}$ are given by

$$
\begin{aligned}
& (29) F=\left(\begin{array}{cc}
-\frac{1}{2}(2 \lambda-u) & 1 \\
-v & \frac{1}{2}(2 \lambda-u)
\end{array}\right) \\
& (30) G=\left(\begin{array}{cc}
-\frac{1}{4}\left((2 \lambda-u) P_{n}+P_{n, x}\right) & \frac{1}{2} P_{n} \\
\frac{1}{2} \sum_{i=0}^{n+1} \lambda^{n+1-i} g_{i}+\frac{1}{2} \lambda^{n} u_{y}-\frac{1}{2} M_{n} & \frac{1}{4}\left((2 \lambda-u) P_{n}+P_{n, x}\right) \\
-\frac{1}{4}\left((2 \lambda-u) P_{n}+P_{n, x}\right)_{x}-\frac{1}{2} v P_{n} &
\end{array}\right)
\end{aligned}
$$

and where $\lambda$ satisfies (26). This is a matrix non-isospectral scattering problem of the form discussed in [11], where the case $n=1$ and $g_{0}=g_{1}=0$ can be found. We note that in the expression for $\mathcal{G}$ there is a cancellation of terms in $u_{n x, y}$ between $M_{n}$ and $P_{n, x x}$ (in the corresponding flow, $u_{t_{n}}=(-1 / 2)^{n} u_{n x, y}+\cdots$ and $\left.v_{t_{n}}=(1 / 2)^{n} v_{n x, y}+\cdots\right)$. Suitable gauge transformations yield other canonical forms of linear problem [11].

Reductions of the system (22) to PDEs in $1+1$ dimensions include nonisospectral deformations of standard $1+1$ DWW flows $\left(\partial_{y}=\partial_{x}\right)$, and also reductions to non-isospectral deformations of inverse DWW flows $\left(\partial_{t_{n}}=0\right)$, all of which are obtained together with corresponding scalar and matrix linear problems.

\section{$\S 3 . \quad$ Reductions in Components}

The hierarchy (22) is a two-component hierarchy of PDEs in $2+1$ dimensions. We now consider reductions of this hierarchy to one component.

We begin by noting that in the case $v=0$ the recursion operator (2) becomes

$$
\mathcal{R}=\frac{1}{2}\left(\begin{array}{cc}
\partial_{x} u \partial_{x}^{-1}-\partial_{x} & 2 \\
0 & u+\partial_{x}
\end{array}\right) .
$$

It then follows that in the reduction $v=0$, the hierarchy (22) becomes the 
(entirely local under $u=w_{x}$ ) scalar hierarchy

$$
u_{t_{n}}=\tilde{\mathcal{R}}^{n} u_{y}+\sum_{i=0}^{n+1} \tilde{\mathcal{R}}^{n+1-i} g_{i}
$$

where the operator $\tilde{\mathcal{R}}=(1 / 2) \partial_{x}\left(u-\partial_{x}\right) \partial_{x}^{-1}$ is the recursion operator of the Burgers hierarchy. Thus we obtain a non-isospectral deformation of a $2+1$ dimensional non-isospectral Burgers hierarchy; special cases of this are discussed in [7] and [11].

Equation (32) is linearized by the transformation $u=-\varphi_{x} / \varphi$ onto

$$
\varphi_{t_{n}}=\left(-\frac{1}{2}\right)^{n} \varphi_{n x, y}-\sum_{i=0}^{n+1}\left(-\frac{1}{2}\right)^{i} g_{n+1-i}\left(i \varphi_{(i-1) x}+x \varphi_{i x}\right) .
$$

We now consider the reduction $u=0$. In this case we find that

$$
\mathcal{R}^{2}=\left(\begin{array}{cc}
\hat{\mathcal{R}} & 0 \\
\frac{1}{4}\left(2 v_{x}+v_{x x} \partial_{x}^{-1}\right) & \hat{\mathcal{R}}
\end{array}\right)
$$

where $\hat{\mathcal{R}}=(1 / 4)\left(\partial_{x}^{2}+4 v+2 v_{x} \partial_{x}^{-1}\right)$ is the recursion operator of the Kortewegde Vries $(\mathrm{KdV})$ hierarchy. Thus we obtain a reduction of the even flows of the hierarchy (22) to

$$
v_{t_{2 n}}=\hat{\mathcal{R}}^{n} v_{y}+\sum_{k=0}^{n} \hat{\mathcal{R}}^{n-k+1} g_{2 k}
$$

where in taking this reduction we have also set $g_{2 k+1}=0$ for $k=0, \ldots, n$. This then gives a non-isospectral deformation of the $2+1$ non-isospectral $\mathrm{KdV}$ hierarchy.

If we now consider reductions to $1+1$ dimensions, then we see that we obtain, in the reduction $v=0$, non-isospectral deformations of both the Burgers hierarchy and of inverse Burgers flows, and in the reduction $u=0$, nonisospectral deformations of both the KdV hierarchy and of inverse KdV flows. We also obtain, for all of these, corresponding scalar and matrix linear problems.

We note that in the special case of the standard $1+1$-dimensional DWW hierarchy, i.e. for $\partial_{y}=\partial_{x}$ and all $g_{i}=0$ in (22), the reductions to the Burgers and KdV hierarchies, which follow from the reductions obtained here, are known. 


\section{$\S 4$. A Generalized $\boldsymbol{P}_{I V}-\boldsymbol{P}_{I I}$ Hierarchy}

We now consider reductions of the hierarchy (22) to systems of ODEs. We take $\partial_{t_{n}}=0$ and $\partial_{y}=\partial_{x}$, which then yields

$$
\mathcal{R}^{n} \mathbf{u}_{x}+\sum_{i=0}^{n+1} \mathcal{R}^{n+1-i} \mathbf{G}_{i}=(0,0)^{T},
$$

where all $g_{i}$ are now constant parameters. Following the approach in [9] we are able to use our non-isospectral scattering problems to obtain linear problems for the hierarchy of ODEs (36). We thus obtain the linear problem

$$
\begin{aligned}
\psi_{x x} & =\left[\left(\lambda-\frac{1}{2} u\right)^{2}+\frac{1}{2} u_{x}-v\right] \psi, \\
\left(\frac{1}{2} \sum_{i=0}^{n+1} \lambda^{n+1-i} g_{i}\right) \psi_{\lambda} & =\left(\lambda^{n}+\frac{1}{2} P_{n}\right) \psi_{x}-\frac{1}{4} P_{n, x} \psi,
\end{aligned}
$$

where we assume that not all $g_{i}$ are zero. Here $P_{n}$ is given by (25), where now

$$
\mathbf{M}_{i}=\mathcal{R}^{i} \mathbf{u}_{x}+\sum_{j=0}^{i+1} \mathcal{R}^{i+1-j} \mathbf{G}_{j}
$$

$\mathbf{M}_{0}=\left(u_{x}+(1 / 2) g_{0}(x u)_{x}+g_{1}, v_{x}+(1 / 2) g_{0}\left(2 v+x v_{x}\right)\right)^{T}$, and as before $\mathbf{M}_{-1}$ $=\left(g_{0}, 0\right)^{T}$.

We also obtain the matrix linear problem

$$
\begin{aligned}
\boldsymbol{\Psi}_{x} & =F \boldsymbol{\Psi}, \\
\left(\frac{1}{2} \sum_{i=0}^{n+1} \lambda^{n+1-i} g_{i}\right) \boldsymbol{\Psi}_{\lambda} & =H \boldsymbol{\Psi}=\left(\lambda^{n} F+G\right) \boldsymbol{\Psi},
\end{aligned}
$$

where now the matrices $\mathcal{F}$ and $\mathcal{G}$ are given by

$$
\begin{aligned}
& \text { (42) } F=\left(\begin{array}{cc}
-\frac{1}{2}(2 \lambda-u) & 1 \\
-v & \frac{1}{2}(2 \lambda-u)
\end{array}\right) \\
& \text { (43) } G=\left(\begin{array}{cc}
-\frac{1}{4}\left((2 \lambda-u) P_{n}+P_{n, x}\right) & \frac{1}{2} P_{n} \\
\frac{1}{2} \sum_{i=0}^{n+1} \lambda^{n+1-i} g_{i}+\frac{1}{2} \lambda^{n} u_{x}-\frac{1}{2} M_{n} & \frac{1}{4}\left((2 \lambda-u) P_{n}+P_{n, x}\right) \\
-\frac{1}{4}\left((2 \lambda-u) P_{n}+P_{n, x}\right)_{x}-\frac{1}{2} v P_{n} &
\end{array}\right)
\end{aligned}
$$

and where $P_{n}$ and $\mathbf{M}_{n}$ are as described above. 
We note that the solution of ODEs using associated linear problems is discussed in [27] and [29]-[31]. Here we present linear problems for hierarchies of ODEs.

In the local case $g_{i}=0, i=0,1, \ldots, n-2$, the hierarchy (36) reads

$$
\begin{aligned}
\mathcal{R}^{n} \mathbf{u}_{x} & +\frac{1}{4} g_{n-1}\left(\begin{array}{c}
\left(x u^{2}-x u_{x}-u\right)_{x}+4 v+2 x v_{x} \\
2 x u_{x} v+2 x u v_{x}+4 v u+x v_{x x}+3 v_{x}
\end{array}\right) \\
+ & \frac{1}{2} g_{n}\left(\begin{array}{c}
(x u)_{x} \\
2 v+x v_{x}
\end{array}\right)+g_{n+1}\left(\begin{array}{l}
1 \\
0
\end{array}\right)=\left(\begin{array}{l}
0 \\
0
\end{array}\right),
\end{aligned}
$$

where we now assume that at least one of $g_{n-1}, g_{n}$, or $g_{n+1}$ is nonzero. We note that in the case $g_{n-1}=g_{n+1}=0$ the hierarchy (44) is the similarity reduction of the DWW hierarchy $\mathbf{u}_{t_{n}}=\mathcal{R}^{n} \mathbf{u}_{x}$ under

$$
u=\frac{U(X)}{\left[\frac{1}{2}(n+1) g_{n} t_{n}\right]^{\frac{1}{n+1}}}, \quad v=\frac{V(X)}{\left[\frac{1}{2}(n+1) g_{n} t_{n}\right]^{\frac{2}{n+1}}}, \quad X=\frac{x}{\left[\frac{1}{2}(n+1) g_{n} t_{n}\right]^{\frac{1}{n+1}}} .
$$

Thus we see that the hierarchy (44) is more general than that which would be obtained using the scaling reduction (45). More general still is the hierarchy (36) which, although written here nonlocally, can always be written locally using suitable auxiliary variables. Notwithstanding, it is the hierarchy (44) that we define here as a generalized $P_{I V}-P_{I I}$ hierarchy; scalar and matrix linear problems follow easily from those given above for (36). As we shall see, this hierarchy has as special cases both a hierarchy having $P_{I V}$ as first member, and also a hierarchy having $P_{I I}$ as first member. Both (36) and (44) are new hierarchies of integrable ODEs.

Reductions in components of (36) follow easily from the discussion in Section 3 . In the case $v=0$ we obtain a hierarchy of linearizable ODEs $\left(\partial_{y}=\partial_{x}\right.$ and $\partial_{t_{n}}=0$ in (32)), and in the case $u=0$ we obtain a generalized $P_{34}$ hierarchy $\left(\partial_{y}=\partial_{x}\right.$ and $\partial_{t_{n}}=0$ in (35)); the standard $P_{34}$ hierarchy is as defined in [32].

We now consider the special case $g_{n-1}=0$ of our generalized $P_{I V}-P_{I I}$ hierarchy (44). It is in this special case that we obtain our $P_{I V}$ and $P_{I I}$ hierarchies, according as to whether $g_{n} \neq 0$ or $g_{n}=0$ respectively. Both of these hierarchies are new. 


\section{§4.1. A $P_{I V}$ hierarchy: $g_{n-1}=0$ and $g_{n} \neq 0$}

In this case the hierarchy (44) has the first integral $\left(I_{n}, J_{n}\right)^{T}$ given by

$$
\left(\begin{array}{c}
I_{n} \\
J_{n}
\end{array}\right)=\left(\begin{array}{c}
L_{n, x}-2 K_{n}-\left(u+2 \frac{g_{n+1}}{g_{n}}\right) L_{n} \\
L_{n} K_{n, x}+v L_{n}^{2}+K_{n}^{2}-L_{n, x} K_{n}+\left(u+2 \frac{g_{n+1}}{g_{n}}\right) L_{n} K_{n}
\end{array}\right)
$$

where

$$
\begin{aligned}
\left(\begin{array}{c}
K_{n} \\
L_{n}
\end{array}\right)= & B_{1}^{-1}\left[\mathcal{R}^{n-1} \mathbf{u}_{x}+g_{n}\left(\begin{array}{l}
1 \\
0
\end{array}\right)+\sum_{i=0}^{n-2}\left(\frac{-g_{n+1}}{g_{n}}\right)^{n-i-1} \mathcal{R}^{i} \mathbf{u}_{x}\right] \\
& +2\left(\frac{-g_{n+1}}{g_{n}}\right)^{n}\left(\begin{array}{l}
0 \\
1
\end{array}\right) .
\end{aligned}
$$

In order to see this we note that

$$
\begin{aligned}
\frac{\partial}{\partial x}\left(\begin{array}{c}
I_{n} \\
J_{n}
\end{array}\right) & =\left(\begin{array}{cc}
-2 & 0 \\
2 K_{n} & 2 L_{n}
\end{array}\right)\left[B_{2}+\frac{g_{n+1}}{g_{n}} B_{1}\right]\left(\begin{array}{l}
K_{n} \\
L_{n}
\end{array}\right) \\
& =\left(\begin{array}{cc}
-2 & 0 \\
2 K_{n} & 2 L_{n}
\end{array}\right)\left[\mathcal{R}^{n} \mathbf{u}_{x}+g_{n} \mathcal{R}\left(\begin{array}{l}
1 \\
0
\end{array}\right)+g_{n+1}\left(\begin{array}{l}
1 \\
0
\end{array}\right)\right] \\
& =\left(\begin{array}{l}
0 \\
0
\end{array}\right) .
\end{aligned}
$$

We define two constants of integration $\alpha_{n}$ and $\beta_{n}$ by

$$
\left(\begin{array}{c}
I_{n} \\
J_{n}
\end{array}\right)=\left(\begin{array}{c}
g_{n}-2 \alpha_{n} \\
\left(\frac{1}{2} g_{n}-\alpha_{n}\right)^{2}-\frac{1}{4} \beta_{n}^{2}
\end{array}\right)
$$

and thus obtain the equivalent system of first integrals

$$
\begin{aligned}
L_{n, x} & =2 K_{n}+\left(u+2 \frac{g_{n+1}}{g_{n}}\right) L_{n}+\left(g_{n}-2 \alpha_{n}\right), \\
K_{n, x} & =\frac{\left(K_{n}+\frac{1}{2} g_{n}-\alpha_{n}\right)^{2}-\frac{1}{4} \beta_{n}^{2}}{L_{n}}-v L_{n} .
\end{aligned}
$$

It is the system (49), or equivalently (50), (51), that is our $P_{I V}$ hierarchy; as we shall see, in the case $n=1$ this system of equations is just the fourth Painlevé equation $P_{I V}$. This $P_{I V}$ hierarchy is different from that presented in [14]. That is, the system (50), (51) represents a new $P_{I V}$ hierarchy. Linear problems for this hierarchy follow from those presented above for (36). 


\section{§4.2. A $P_{I I}$ hierarchy: $g_{n-1}=0$ and $g_{n}=0$}

In this case each component of our hierarchy (44) integrates immediately to give

$$
\left(\begin{array}{cc}
\partial_{x}^{-1} & 0 \\
0 & \partial_{x}^{-1}
\end{array}\right) \mathcal{R}^{n} \mathbf{u}_{x}+g_{n+1}\left(\begin{array}{l}
x \\
0
\end{array}\right)=\left(\begin{array}{l}
\gamma_{n} \\
\delta_{n}
\end{array}\right)
$$

where $\gamma_{n}$ and $\delta_{n}$ are two constants of integration, and where we now assume that $g_{n+1} \neq 0$; this assumption then allows us to take $\gamma_{n}=0$.

It is this hierarchy that is our $P_{I I}$ hierarchy; as we shall see, in the case $n=1$ this system of equations is just the second Painlevé equation $P_{I I}$. We note, however, that higher members of this hierarchy are different from those of the $P_{I I}$ hierarchy presented in [26], [27]. That is, the system (52) represents a new $P_{I I}$ hierarchy. Again, linear problems for this hierarchy follow from those presented above for (36).

Remark 1. It is a simple matter to generalise our PDE and ODE hierarchies to include lower order DWW flows. Thus corresponding to the local case (44) we obtain

$$
\mathcal{R}^{n} \mathbf{u}_{x}+\sum_{i=0}^{n-2} c_{i} \mathcal{R}^{i} \mathbf{u}_{x}+g_{n-1} \mathcal{R}^{2}\left(\begin{array}{l}
1 \\
0
\end{array}\right)+g_{n} \mathcal{R}\left(\begin{array}{l}
1 \\
0
\end{array}\right)+g_{n+1}\left(\begin{array}{l}
1 \\
0
\end{array}\right)=\left(\begin{array}{l}
0 \\
0
\end{array}\right)
$$

where without loss of generality we have taken $c_{n-1}=0$, together with corresponding scalar and matrix linear problems. We also obtain corresponding generalizations of our $P_{I V}$ and $P_{I I}$ hierarchies, again with their corresponding linear problems.

Remark 2. Thus far we have assumed that not all $g_{i}$ are zero. In the case where all $g_{i}$ vanish then the coefficients in $\lambda$ of the determinant of $H$ in (41) give constants of integration of the system (44). Similarly for the corresponding linear problem of (52) in the case $g_{n+1}=0$, and for the more general $P_{I V}-P_{I I}$ and $P_{I I}$ hierarchies discussed in Remark 1 above. (Note that in any linear problem we always replace higher derivatives of $u$ and $v$ using the corresponding equations; see [13], [14].) 


\section{$\S 5$. Examples}

\section{$\S 5.1 . \quad n=1$}

In the case $n=1$ we obtain the system of PDEs (9), or equivalently (10) and (11). Corresponding to (44) we obtain

$$
\mathcal{R} \mathbf{u}_{x}+g_{0} \mathcal{R}^{2}\left(\begin{array}{l}
1 \\
0
\end{array}\right)+g_{1} \mathcal{R}\left(\begin{array}{l}
1 \\
0
\end{array}\right)+g_{2}\left(\begin{array}{l}
1 \\
0
\end{array}\right)=\left(\begin{array}{l}
0 \\
0
\end{array}\right),
$$

i.e.

$$
\frac{1}{2}\left(2 v+u^{2}-u_{x}\right)_{x}+\frac{1}{4} g_{0}\left(\left(x u^{2}-x u_{x}-u\right)_{x}+4 v+2 x v_{x}\right)+\frac{1}{2} g_{1}(x u)_{x}+g_{2}=0
$$

$\frac{1}{2}\left(2 u v+v_{x}\right)_{x}+\frac{1}{4} g_{0}\left(2 x u_{x} v+2 x u v_{x}+4 v u+x v_{x x}+3 v_{x}\right)+\frac{1}{2} g_{1}\left(2 v+x v_{x}\right)=0$ together with the matrix linear problem (40), (41) with $F$ and $H$ given by

$$
\begin{aligned}
& F=\left(\begin{array}{cc}
-\frac{1}{2}(2 \lambda-u) & 1 \\
-v & \frac{1}{2}(2 \lambda-u)
\end{array}\right), \\
& H=\left(\begin{array}{cc}
-\lambda^{2}+\frac{1}{4}\left(u^{2}-u_{x}\right)+\frac{1}{4} g_{1}(x u & \lambda+\frac{1}{2} u+\frac{1}{2} x g_{1} \\
-2 x \lambda-1)+\frac{1}{8} g_{0}\left(x u^{2}\right. & +\frac{1}{4} g_{0}(x u+2 x \lambda) \\
\left.-x u_{x}-u-4 x \lambda^{2}-2 \lambda\right) & \\
-\lambda v-\frac{1}{2}\left(v_{x}+u v\right)-\frac{1}{2} x g_{1} v & \lambda^{2}-\frac{1}{4}\left(u^{2}-u_{x}\right)-\frac{1}{4} g_{1}(x u \\
-\frac{1}{4} g_{0}\left(x v_{x}+x u v+2 x \lambda v+2 v\right) & -2 x \lambda-1)-\frac{1}{8} g_{0}\left(x u^{2}\right. \\
& \left.-x u_{x}-u-4 x \lambda^{2}-2 \lambda\right)
\end{array}\right) .
\end{aligned}
$$

This system of ODEs is the first member of our generalized $P_{I V}-P_{I I}$ hierarchy.

In the case $g_{0}=0$ and $g_{1} \neq 0$ we obtain first integrals as in Section 4.1, with $K_{1}$ and $L_{1}$ given by

$$
\left(\begin{array}{c}
K_{1} \\
L_{1}
\end{array}\right)=\left(\begin{array}{c}
v \\
u+g_{1} x-2\left(g_{2} / g_{1}\right)
\end{array}\right) .
$$

Corresponding to (50), (51) we thus obtain

$$
\begin{aligned}
& u_{x}=2 v+u^{2}+g_{1} x u+2 g_{2} x-2 \alpha_{1}-4\left(g_{2} / g_{1}\right)^{2}, \\
& v_{x}=\frac{\left[v-\alpha_{1}+\frac{1}{2} g_{1}\right]^{2}-\frac{1}{4} \beta_{1}^{2}}{\left[u+g_{1} x-2\left(g_{2} / g_{1}\right)\right]}-v\left[u+g_{1} x-2\left(g_{2} / g_{1}\right)\right],
\end{aligned}
$$


where $\alpha_{1}$ and $\beta_{1}$ are two independent constants of integration. Solving the first of these for $v$,

$$
v=\frac{1}{2}\left[u_{x}-u^{2}-g_{1} x u-2 g_{2} x+2 \alpha_{1}+4\left(g_{2} / g_{1}\right)^{2}\right],
$$

and substituting into the second then yields a scalar second order ODE in $u$ which, under the change of variables

$$
\begin{aligned}
u(x) & =y(z)-g_{1} z-2\left(g_{2} / g_{1}\right) \\
x & =z+4\left(g_{2} / g_{1}^{2}\right)
\end{aligned}
$$

becomes

$$
y_{z z}=\frac{1}{2} \frac{y_{z}^{2}}{y}+\frac{3}{2} y^{3}-2 g_{1} z y^{2}+2\left[\left(g_{1}^{2} z^{2} / 4\right)-\alpha_{1}\right] y-\frac{1}{2} \frac{\beta_{1}^{2}}{y} .
$$

Setting $g_{1}=-2$, which can be done without loss of generality for $g_{1} \neq 0$, gives

$$
y_{z z}=\frac{1}{2} \frac{y_{z}^{2}}{y}+\frac{3}{2} y^{3}+4 z y^{2}+2\left(z^{2}-\alpha_{1}\right) y-\frac{1}{2} \frac{\beta_{1}^{2}}{y},
$$

which is just the fourth Painlevé equation $P_{I V}$. Thus we see that (49), or equivalently (50), (51), is indeed a $P_{I V}$ hierarchy. Corresponding to (40), (41) we obtain for (66) the linear problem

$$
\begin{aligned}
& \text { (67) } F=\left(\begin{array}{cc}
\frac{1}{2}\left(y-z g_{1}-2 \mu\right) & 1 \\
\frac{1}{2}\left(y^{2}-y_{z}-z g_{1} y+g_{1}-2 \alpha_{1}\right) & -\frac{1}{2}\left(y-z g_{1}-2 \mu\right)
\end{array}\right), \\
& \text { (68) } H=\left(\begin{array}{cc}
\frac{1}{4}\left(y^{2}-y_{z}-z g_{1} y-2 z \mu g_{1}-4 \mu^{2}\right) & \frac{1}{2}(2 \mu+y) \\
\frac{1}{8}\left[2 y y_{z}-\frac{y_{z}^{2}}{y}-y^{3}+4 \mu\left(y^{2}-y_{z}\right)\right. & -\frac{1}{4}\left(y^{2}-y_{z}-z g_{1} y\right. \\
-z^{2} g_{1}^{2} y+2 g_{1}\left(z y^{2}-z y_{z}+2 \mu\right. & \left.-2 z \mu g_{1}-4 \mu^{2}\right) \\
\left.-2 z \mu y)+\frac{\beta_{1}^{2}}{y}-8 \alpha_{1} \mu\right] &
\end{array}\right),
\end{aligned}
$$

where we have set $\lambda=\mu-\left(g_{2} / g_{1}\right)$ [this change also needs to be made in (41)].

In the case $g_{0}=g_{1}=0$ we obtain corresponding to (52) the system

$$
\begin{array}{r}
v+\frac{1}{2}\left(u^{2}-u_{x}\right)+x g_{2}-\gamma_{1}=0, \\
u v+\frac{1}{2} v_{x}-\delta_{1}=0,
\end{array}
$$


where $\gamma_{1}$ and $\delta_{1}$ are two independent constants of integration. This system is equivalent to the second order ODE

$$
u_{x x}=2 u^{3}+4 x g_{2} u-4 \gamma_{1} u+2\left(g_{2}+2 \delta_{1}\right),
$$

which for $g_{2} \neq 0$ (in which case we can take $\gamma_{1}=0$ ) is just the second Painlevé equation $P_{I I}$. Thus we see that our hierarchy (52) is indeed a $P_{I I}$ hierarchy. Corresponding to (40), (41) we obtain for (71) the linear problem

$$
\begin{aligned}
& F=\left(\begin{array}{cc}
-\lambda+\frac{1}{2} u & 1 \\
\frac{1}{2}\left(u^{2}-u_{x}\right)+x g_{2}-\gamma_{1} & \lambda-\frac{1}{2} u
\end{array}\right), \\
& H=\left(\begin{array}{cc}
\frac{1}{4}\left(u^{2}-u_{x}-4 \lambda^{2}\right) & \lambda+\frac{1}{2} u \\
\frac{1}{4}\left[u u_{x}-u^{3}+2 \lambda\left(u^{2}-u_{x}\right)+2 \gamma_{1} u\right. & -\frac{1}{4}\left(u^{2}-u_{x}-4 \lambda^{2}\right) \\
\left.+2 x g_{2}(2 \lambda-u)-4 \gamma_{1} \lambda-4 \delta_{1}\right] &
\end{array}\right) .
\end{aligned}
$$

We note that in the case where all $g_{i}=0$ the determinant of $H$ gives for both (54) and (71) a quartic in $\lambda$ with coefficients constants of integration of the equations.

\section{$\S 5.2 . \quad n=2$}

In the case $n=2$ we obtain the system of PDEs

$$
\begin{aligned}
w_{x t_{2}}= & \frac{1}{4}\left[\left(w_{x}^{2} w_{y}-w_{x x} w_{y}-2 w_{x} w_{x y}+w_{x x y}\right)_{x}\right. \\
& \left.+2\left(z_{x x} w_{y}+2 z_{x} w_{x y}+2 w_{x} z_{x y}+w_{x x} z_{y}\right)\right] \\
& +\frac{1}{4} g_{1}\left(\left(x w_{x}^{2}-x w_{x x}-w_{x}\right)_{x}+4 z_{x}+2 x z_{x x}\right)+\frac{1}{2} g_{2}\left(x w_{x}\right)_{x}+g_{3}
\end{aligned}
$$

$$
\begin{aligned}
z_{x t_{2}}= & \frac{1}{4}\left[2 z_{x} w_{x x} w_{y}+4 z_{x} w_{x} w_{x y}+2 z_{x x} w_{x y}+z_{x x x} w_{y}+w_{x x} z_{x y}+w_{x} z_{x x y}\right. \\
& \left.+4 z_{x} z_{x y}+2 z_{x x} z_{y}+w_{x}^{2} z_{x y}+2 w_{x} w_{y} z_{x x}+w_{x} z_{x x y}+z_{x x x y}\right] \\
& +\frac{1}{4} g_{1}\left(2 x w_{x x} z_{x}+2 x w_{x} z_{x x}+4 w_{x} z_{x}+x z_{x x x}+3 z_{x x}\right)+\frac{1}{2} g_{2}\left(2 z_{x}+x z_{x x}\right)
\end{aligned}
$$

where we have set $u=w_{x}$ and $v=z_{x}$, together with corresponding scalar and matrix linear problems. In the above system, and throughout this section, we have set $g_{0}=0$ in order to consider only the local case of our PDEs and ODEs 
(that is, without the use of auxiliary variables). To the above system we can readily add terms $c u_{x}$ and $c v_{x}$ so that we obtain as ODE reduction the system

$$
\mathcal{R}^{2} \mathbf{u}_{x}+c \mathbf{u}_{x}+g_{1} \mathcal{R}^{2}\left(\begin{array}{l}
1 \\
0
\end{array}\right)+g_{2} \mathcal{R}\left(\begin{array}{l}
1 \\
0
\end{array}\right)+g_{3}\left(\begin{array}{l}
1 \\
0
\end{array}\right)=\left(\begin{array}{l}
0 \\
0
\end{array}\right),
$$

as explained in Remark 1 (and where we have relabelled $c_{0}$ as $c$ ).

This system of ODEs is

(77) $\frac{1}{4}\left(u_{x x}-3 u u_{x}+u^{3}+6 u v\right)_{x}+c u_{x}$

$$
+\frac{1}{4} g_{1}\left(\left(x u^{2}-x u_{x}-u\right)_{x}+4 v+2 x v_{x}\right)+\frac{1}{2} g_{2}(x u)_{x}+g_{3}=0,
$$

(78) $\frac{1}{4}\left(v_{x x}+3 v^{2}+3 u v_{x}+3 u^{2} v\right)_{x}+c v_{x}$

$$
+\frac{1}{4} g_{1}\left(2 x u_{x} v+2 x u v_{x}+4 v u+x v_{x x}+3 v_{x}\right)+\frac{1}{2} g_{2}\left(2 v+x v_{x}\right)=0,
$$

and is (with $c=0$ ) the second member of our generalized $P_{I V}-P_{I I}$ hierarchy.

We obtain for this system the matrix linear problem (40), (41) with $F$ and $G$ given by

$$
\begin{aligned}
& F=\left(\begin{array}{cc}
-\lambda+\frac{1}{2} u & 1 \\
-v & \lambda-\frac{1}{2} u
\end{array}\right), \\
& H=\left(\begin{array}{cc}
H_{11} & H_{12} \\
H_{21} & -H_{11}
\end{array}\right),
\end{aligned}
$$

where

$$
\begin{aligned}
H_{11}= & -\lambda^{3}+c\left(\frac{1}{2} u-\lambda\right)+\frac{1}{8}\left(u_{x x}-2 v_{x}+u^{3}-3 u u_{x}+2 u v-4 \lambda v\right) \\
& +\frac{1}{4} g_{2}(x u-2 x \lambda-1)-\frac{1}{8} g_{1}\left(4 x \lambda^{2}+2 \lambda+u-x u^{2}+x u_{x}\right), \\
H_{12}= & \lambda^{2}+\frac{1}{2} \lambda u+c+\frac{1}{4}\left(u^{2}-u_{x}+2 v\right)+\frac{1}{2} x g_{2}+\frac{1}{4} g_{1}(2 x \lambda+x u), \\
H_{21}= & -\lambda^{2} v-\frac{1}{2} \lambda\left(u v+v_{x}\right)-c v-\frac{1}{4}\left(v_{x x}+v u_{x}+2 u v_{x}+u^{2} v+2 v^{2}\right) \\
& -\frac{1}{2} x g_{2} v-\frac{1}{4} g_{1}\left(2 x \lambda v+2 v+x u v+x v_{x}\right) .
\end{aligned}
$$

In the case $g_{1}=0$ and $g_{2} \neq 0$ we obtain first integrals as in Section 4.1, with $K_{2}$ and $L_{2}$ given by

$$
\left(\begin{array}{c}
K_{2} \\
L_{2}
\end{array}\right)=\left(\begin{array}{c}
u v+\frac{1}{2} v_{x}-\left(g_{3} / g_{2}\right) v \\
v+\frac{1}{2} u^{2}-\frac{1}{2} u_{x}+g_{2} x-\left(g_{3} / g_{2}\right) u+2\left(g_{3} / g_{2}\right)^{2}+2 c
\end{array}\right) .
$$


Corresponding to (50), (51) we thus obtain

$u_{x x}=3 u u_{x}-u^{3}-6 u v-2 g_{2} x u-4 g_{3} x+4 \alpha_{2}-8\left(g_{3} / g_{2}\right)^{3}-4 c\left(u+2\left(g_{3} / g_{2}\right)\right)$,

$$
\begin{aligned}
v_{x x}= & 2\left(\frac{\left[u v+\frac{1}{2} v_{x}-\left(g_{3} / g_{2}\right) v-\alpha_{2}+\frac{1}{2} g_{2}\right]^{2}-\frac{1}{4} \beta_{2}^{2}}{v+\frac{1}{2} u^{2}-\frac{1}{2} u_{x}+g_{2} x-\left(g_{3} / g_{2}\right) u+2\left(g_{3} / g_{2}\right)^{2}+2 c}\right) \\
& -2 v\left(v+\frac{1}{2} u^{2}-\frac{1}{2} u_{x}+g_{2} x-\left(g_{3} / g_{2}\right) u+2\left(g_{3} / g_{2}\right)^{2}+2 c\right) \\
& -2(u v)_{x}+2\left(g_{3} / g_{2}\right) v_{x},
\end{aligned}
$$

where $\alpha_{2}$ and $\beta_{2}$ are two independent constants of integration. Solving the first of these for $v$,

$$
\begin{aligned}
v= & -\frac{1}{6 u}\left[u_{x x}-3 u u_{x}+u^{3}+2 g_{2} x u+4 g_{3} x\right. \\
& \left.-4 \alpha_{2}+8\left(g_{3} / g_{2}\right)^{3}+4 c\left(u+2\left(g_{3} / g_{2}\right)\right)\right],
\end{aligned}
$$

and substituting into the second then yields a scalar fourth order ODE in $u$ which is too long to give here. The system (85), (86), or equivalently this scalar ODE in $u$, is the second member of our new $P_{I V}$ hierarchy. This system may also be written as (where the first of these defines $w$ )

$$
\begin{aligned}
u_{x x}= & 2 u^{3}-6\left(g_{3} / g_{2}\right) u^{2}+4\left[2 c+3\left(g_{3} / g_{2}\right)^{2}\right] u+4 g_{2} x u-4 g_{3} x+w \\
& +4\left[\alpha_{2}-2 c\left(g_{3} / g_{2}\right)-2\left(g_{3} / g_{2}\right)^{3}\right] \\
w_{x x}= & \frac{1}{2} \frac{w_{x}^{2}}{w}+\frac{u_{x}}{u} w_{x}+\frac{4}{3 u} w^{2}-\frac{3 u_{x}^{2}}{2 u^{2}} w+\frac{7}{2} u^{2} w-6\left(g_{3} / g_{2}\right) u w \\
& +4\left(\alpha_{2}-g_{3} x-2 c\left(g_{3} / g_{2}\right)-2\left(g_{3} / g_{2}\right)^{3}\right) \frac{w}{u}-18 \beta_{2}^{2} \frac{u^{2}}{w} \\
& +6\left(g_{2} x+2 c+3\left(g_{3} / g_{2}\right)^{2}\right) w
\end{aligned}
$$

corresponding to which we have a matrix linear problem (40), (41) with

$$
\begin{aligned}
F & =\left(\begin{array}{cc}
-\lambda+\frac{1}{2} u & 1 \\
2 c+g_{2} x+2\left(g_{3} / g_{2}\right)^{2}-\left(g_{3} / g_{2}\right) u & \lambda-\frac{1}{2} u \\
+(1 / 2)\left(u^{2}-u^{\prime}\right)+(w / 6 u) &
\end{array}\right), \\
H & =\left(\begin{array}{cc}
H_{11} & H_{12} \\
H_{21} & -H_{11}
\end{array}\right),
\end{aligned}
$$

where

$$
H_{11}=\left(1 /\left(24 g_{2}^{2} u^{2}\right)\right)\left[24 \lambda g_{3}^{2} u^{2}-24 \lambda^{3} g_{2}^{2} u^{2}-12 \lambda g_{2} g_{3} u^{3}-12 g_{3}^{2} u^{3}\right.
$$




$$
\begin{aligned}
& +6 \lambda g_{2}^{2} u^{4}+6 g_{2} g_{3} u^{4}+2 \lambda g_{2}^{2} u w-g_{2}^{2} u^{2} w-6 \lambda g_{2}^{2} u^{2} u_{x} \\
& \left.-6 g_{2} g_{3} u^{2} u_{x}-g_{2}^{2} w u_{x}+g_{2}^{2} u w_{x}\right], \\
(93) \quad H_{12}= & \left(1 /\left(12 g_{2}^{2} u\right)\right)\left[12 \lambda^{2} g_{2}^{2} u-12 g_{3}^{2} u+6 \lambda g_{2}^{2} u^{2}+6 g_{2} g_{3} u^{2}-g_{2}^{2} w\right], \\
(94) \quad H_{21}= & \left(1 /\left(48 g_{2}^{3} u^{3} w\right)\right)\left[96 c \lambda^{2} g_{2}^{2} u^{3} w-36 \beta_{2}^{2} g_{2}^{3} u^{4}-48 \alpha_{2} \lambda g_{2}^{3} u^{3} w\right. \\
& +24 \lambda g_{2}^{4} u^{3} w+48 \lambda^{2} g_{2}^{4} x u^{3} w-48 \alpha_{2} g_{2}^{2} g_{3} u^{3} w+96 c \lambda g_{2}^{2} g_{3} u^{3} w \\
& +24 g_{2}^{3} g_{3} u^{3} w+48 \lambda g_{2}^{3} g_{3} x u^{3} w+96 \lambda^{2} g_{2} g_{3}^{2} u^{3} w+96 \lambda g_{3}^{3} u^{3} w \\
& -48 c \lambda g_{2}^{3} u^{4} w-24 \lambda g_{2}^{4} x u^{4} w-48 c g_{2}^{2} g_{3} u^{4} w-48 \lambda^{2} g_{2}^{2} g_{3} u^{4} w \\
& -24 g_{2}^{3} g_{3} x u^{4} w-96 \lambda g_{2} g_{3}^{2} u^{4} w-48 g_{3}^{3} u^{4} w+24 \lambda^{2} g_{2}^{3} u^{5} w \\
& +48 \lambda g_{2}^{2} g_{3} u^{5} w+24 g_{2} g_{3}^{2} u^{5} w-12 \lambda g_{2}^{3} u^{6} w-12 g_{2}^{2} g_{3} u^{6} w \\
& +8 \lambda^{2} g_{2}^{3} u^{2} w^{2}-4 g_{2} g_{3}^{2} u^{2} w^{2}-8 \lambda g_{2}^{3} u^{3} w^{2}-4 g_{2}^{2} g_{3} u^{3} w^{2} \\
& +g_{2}^{3} u^{4} w^{2}-24 \lambda^{2} g_{2}^{3} u^{3} w u_{x}-24 \lambda g_{2}^{2} g_{3} u^{3} w u_{x}+12 \lambda g_{2}^{3} u^{4} w u_{x} \\
& +12 g_{2}^{2} g_{3} u^{4} w u_{x}-4 \lambda g_{2}^{3} u w^{2} u_{x}+2 g_{2}^{3} u^{2} w^{2} u_{x}+g_{2}^{3} w^{2} u_{x}^{2} \\
& \left.+4 \lambda g_{2}^{3} u^{2} w w_{x}-2 g_{2}^{3} u^{3} w w_{x}-2 g_{2}^{3} u w u_{x} w_{x}+g_{2}^{3} u^{2} w_{x}^{2}\right] .
\end{aligned}
$$

The Painlevé analysis of our fourth order ODE in $u$ is of particular interest since it presents a previously unseen difficulty for Painlevé classification; this will be discussed in a later paper.

In the case $g_{1}=g_{2}=0$, we obtain corresponding to (52) the system

$$
\begin{aligned}
\frac{1}{4}\left(u_{x x}-3 u u_{x}+u^{3}+6 u v\right)+c u+g_{3} x-\gamma_{2} & =0, \\
\frac{1}{4}\left(v_{x x}+3 v^{2}+3 u v_{x}+3 u^{2} v\right)+c v-\delta_{2} & =0,
\end{aligned}
$$

where $\gamma_{2}$ and $\delta_{2}$ are two independent constants of integration. Solving the first of these for $v$,

$$
v=-\frac{1}{6 u}\left[u_{x x}-3 u u_{x}+u^{3}+4 c u+4 g_{3} x-4 \gamma_{2}\right],
$$

and substituting into the second, we see that this system is equivalent to the fourth order ODE

$$
\begin{aligned}
u_{x x x x}= & 2 \frac{u_{x x x} u_{x}}{u}+\frac{3}{2} \frac{u_{x x}^{2}}{u}-2 \frac{u_{x x} u_{x}^{2}}{u^{2}}+5 u^{2} u_{x x}-8\left(\gamma_{2}-g_{3} x\right) \frac{u_{x x}}{u} \\
& +\frac{5}{2} u u_{x}^{2}+8\left(\gamma_{2}-g_{3} x\right) \frac{u_{x}^{2}}{u^{2}}+8 g_{3} \frac{u_{x}}{u}-\frac{5}{2} u^{5}-12 c u^{3} \\
& +8\left(\gamma_{2}-g_{3} x\right) u^{2}-4\left(2 c^{2}+6 \delta_{2}+3 g_{3}\right) u+8 \frac{\left(\gamma_{2}-g_{3} x\right)^{2}}{u},
\end{aligned}
$$

where in the case $g_{3} \neq 0$ we can set $\gamma_{2}=0$. The system (95), (96), or equivalently this scalar ODE in $u$, is the second member of our new $P_{I I}$ hierarchy. 
Corresponding to the ODE (98) we have the matrix linear problem (40), (41) with

$$
\begin{aligned}
F & =\left(\begin{array}{cc}
-\lambda+\frac{1}{2} u & 1 \\
4\left(g_{3} x-\gamma_{2}\right)+4 c u & \lambda-\frac{1}{2} u \\
+u_{x x}-3 u u_{x}+u^{3} &
\end{array}\right), \\
H & =\left(\begin{array}{cc}
H_{11} & H_{12} \\
H_{21} & -H_{11}
\end{array}\right),
\end{aligned}
$$

where

$$
\begin{aligned}
(101) H_{11}= & \left(1 /\left(24 u^{2}\right)\right)\left[4 g_{3} u-8 \lambda \gamma_{2} u+8 \lambda g_{3} x u+4 \gamma_{2} u^{2}-16 \lambda c u^{2}\right. \\
& -24 \lambda^{3} u^{2}-4 g_{3} x u^{2}+8 c u^{3}+2 \lambda u^{4}+2 u^{5}+4 \gamma_{2} u_{x}-4 g_{3} x u_{x} \\
& \left.-6 \lambda u^{2} u_{x}-4 u^{3} u_{x}+2 \lambda u u_{x x}-u^{2} u_{x x}-u_{x} u_{x x}+u u_{x x x}\right], \\
(102) \quad H_{12}= & (1 /(12 u))\left[4 \gamma_{2}-4 g_{3} x+8 c u+12 \lambda^{2} u+6 \lambda u^{2}+2 u^{3}-u_{x x}\right], \\
(103) \quad H_{21}= & \left(1 /\left(144 u^{2}\right)\right)\left[16 \gamma_{2}^{2}-32 \gamma_{2} g_{3} x+16 g_{3}^{2} x^{2}-32 c \gamma_{2} u-96 \lambda^{2} \gamma_{2} u\right. \\
& +48 \lambda g_{3} u+32 c g_{3} x u+96 \lambda^{2} g_{3} x u+16 c^{2} u^{2}-144 \delta_{2} u^{2} \\
& -48 \lambda \gamma_{2} u^{2}+96 \lambda^{2} c u^{2}-24 g_{3} u^{2}+48 \lambda g_{3} x u^{2}+40 \gamma_{2} u^{3}+48 \lambda c u^{3} \\
& -40 g_{3} x u^{3}-40 c u^{4}+24 \lambda^{2} u^{4}+12 \lambda u^{5}-11 u^{6}+48 \lambda \gamma_{2} u_{x} \\
& -48 \lambda g_{3} x u_{x}-24 \gamma_{2} u u_{x}+24 g_{3} x u u_{x}-72 \lambda^{2} u^{2} u_{x}-12 \lambda u^{3} u_{x} \\
& +24 u^{4} u_{x}-9 u^{2} u_{x}^{2}-8 \gamma_{2} u_{x x}+8 g_{3} x u_{x x}+8 c u u_{x x}+24 \lambda^{2} u u_{x x} \\
& -24 \lambda u^{2} u_{x x}+8 u^{3} u_{x x}-12 \lambda u_{x} u_{x x}+6 u u_{x} u_{x x}+u_{x x}^{2} \\
& \left.+12 \lambda u u_{x x x}-6 u^{2} u_{x x x}\right] .
\end{aligned}
$$

We believe that it is likely that (for $g_{3} \neq 0$ ) this fourth order ODE defines a new transcendent; we note in particular that this ODE is not of polynomial form.

Defining $\varepsilon$ by $2 c^{2}+6 \delta_{2}+3 g_{3}=\varepsilon / 4$ and assuming $g_{3} \neq 0$, so that we may take $\gamma_{2}=0$, we note that this ODE takes the particularly elegant form

$$
\begin{aligned}
u_{x x x x}= & 2 \frac{u_{x x x} u_{x}}{u}+\frac{3}{2} \frac{u_{x x}^{2}}{u}-2 \frac{u_{x x} u_{x}^{2}}{u^{2}}+5 u^{2} u_{x x}+8 g_{3} x \frac{u_{x x}}{u}+\frac{5}{2} u u_{x}^{2} \\
& -8 g_{3} x \frac{u_{x}^{2}}{u^{2}}+8 g_{3} \frac{u_{x}}{u}-\frac{5}{2} u^{5}-12 c u^{3}-8 g_{3} x u^{2}-\varepsilon u+8 \frac{g_{3}^{2} x^{2}}{u}
\end{aligned}
$$

for which the linear problem follows from that given above.

We note that in the case where all $g_{i}=0$ the determinant of $H$ gives for both (76), (98) a polynomial in $\lambda$ of degree six with coefficients constants of integration of the equations. 


\section{$\S 6 . \quad$ Conclusions}

We have given a generalized $2+1$-dimensional non-isospectral DWW hierarchy, together with underlying scalar and matrix linear problems. For this hierarchy we have discussed reductions to PDEs in $1+1$ dimensions, reductions in components, and also reductions to ODEs. In the case of reductions to ODEs we have obtained a new generalized $P_{I V}-P_{I I}$ hierarchy, and as special cases of this a new $P_{I V}$ hierarchy and a new $P_{I I}$ hierarchy. For all of these we have corresponding scalar and matrix linear problems. It is worth noting the advantages of our approach over standard Painlevé classification. First, in the non-local case, recovering our hierarchies requires the use of auxiliary variables. Second, it appears unlikely that one of the ODEs obtained here can be obtained by classification based on the standard Painlevé test (as will be discussed in a later paper). Third, to date the only Painlevé classifications of ODEs appearing in the literature are for scalar equations rather than for systems, and in the case of ODEs of order greater than two are for equations of polynomial type. Here we obtain new hierarchies of systems of ODEs, and also higher order scalar ODEs of rational type; we draw particular attention to the simplest of our new examples (104). This then shows the strengths of the methodology developed in [13], [14].

\section{Acknowledgements}

The research of PRG and AP was supported in part by the DGICYT under contract PB98-0262. NJ's research was supported by the Australian Research Council under Grant \#A69803721 and Fellowship \#F69700172. AP thanks the Ministry of Education and Culture of Spain for a post-doctoral fellowship. He also thanks Professor Cerveró for his kind hospitality during his stay in Salamanca.

\section{References}

[1] Calogero, F., A method to generate solvable nonlinear evolution equations, Lett. Nuovo Cim., 14 (1975), 443-447.

[2] Zakharov, V. E., The inverse scattering method, in Solitons, eds. by R. K. Bullough and P. J. Caudrey, Springer, Berlin, 1980.

[3] Calogero, F. and Degasperis, A., Spectral Transform and Solitons I, North-Holland, Amsterdam-New York-Oxford, 1982.

[4] Levi, D., Hierarchies of integrable equations obtained as nonisospectral (in $x$ and $t$ ) deformations of the Schrödinger spectral problem, Phys. Lett. A, 119 (1987), 453-456.

[5] Burtsev, S. P., Zakharov, V. E. and Mikhailov, A. V., Inverse scattering method with variable spectral parameter, Teoret. i Matem. Fizika, 70 (1987), 323-341. 
[6] Levi, D. and Ragnisco, O., Non-isospectral deformations and Darboux transformations for the third order spectral problem, Inverse Problems, 4 (1988), 815-828.

[7] Bogoyavlenskii, O. I., Overturning solitons in two-dimensional integrable equations, $U s$ pekhi Mat. Nauk, 45 (1990), 17-77; Russian Math. Surveys, 45 (1990), 1-86.

[8] Strachan, I. A. B., Wave solutions of a 2+1-dimensional generalization of the nonlinear Schrödinger equation, Inverse Problems, 8 (1992), L21-L27.

[9] Levi, D., Ragnisco, O. and Rodriguez, M. A., On non-isospectral flows, Painlevé equations and symmetries of differential and difference equations, Teoret. i Matem. Fizika, 93 (1992), 473-480; Theor. Math. Phys., 93 (1993), 1409-1414.

[10] Li, Yi-shen and Zhang, You-jin, Symmetries of a $2+1$-dimensional breaking soliton equation, J. Phys. A, 26 (1993), 7487-7494.

[11] Pickering, A., The singular manifold method revisited, J. Math. Phys., 37 (1996), 18941927.

[12] Gordoa, P. R. and Pickering, A., Bäcklund transformations for two new integrable partial differential equations, Europhys. Lett., 47 (1999), 21-24.

[13] - Non-isospectral scattering problems: a key to integrable hierarchies, J. Math. Phys., 40 (1999), 5749-5786.

[14] $\longrightarrow$, On a new non-isospectral variant of the Boussinesq hierarchy, J. Phys. A, 33 (2000), 557-567.

[15] Gordoa, P. R., Symmetries, exact solutions, and nonlinear superposition formulas for two integrable partial differential equations, J. Math. Phys., 41 (2000), 4713-4731.

[16] Estévez, P. G. and Hernáez, G. A., Non-isospectral problem in $(2+1)$ dimensions, J. Phys. A, 33 (2000), 2131-2143.

[17] - Lax pair, Darboux transformations and solitonic solutions for a $2+1$ dimensional nonlinear Schrödinger equation, J. Nonlinear Math. Phys., to appear, 2000.

[18] Broer, L. J. F., Approximate equations for long water waves, Appl. Sci. Res., 31 (1975), 377-395.

[19] Kaup, D. J., Finding eigenvalue problems for solving nonlinear evolution equations, Prog. Theor. Phys., 54 (1975), 72-78.

[20] - A higher-order water-wave equation and the method for solving it, Prog. Theor. Phys., 54 (1975), 396-408.

[21] Jaulent, M. and Miodek, J., Nonlinear evolution equations associated with energydependent Schrödinger potentials, Lett. Math. Phys., 1 (1976), 243-250.

[22] Matveev, V. B. and Yavor, M. I., Solutions presque périodiques et à $N$ solitons de l'équation hydrodynamique non linéaire de Kaup, Ann. Inst. H. Poincaré, 31 (1979), 25-41.

[23] Martínez, Alonso, L., Schrödinger spectral problems with energy-dependent potentials as sources of nonlinear Hamiltonian evolution equations, J. Math. Phys., 21 (1980), $2342-2349$

[24] Kupershmidt, B. A., Mathematics of dispersive water waves, Comm. Math. Phys., 99 (1985), 51-73.

[25] Sachs, R. L., On the integrable variant of the Boussinesq system: Painlevé property, rational solutions, a related many-body system, and equivalence with the AKNS hierarchy, Physica D, 30 (1988), 1-27.

[26] Airault, H., Rational solutions of Painlevé equations, Stud. Appl. Math., 61 (1979), $31-53$.

[27] Flaschka, H. and Newell, A. C., Monodromy and spectrum preserving deformations, I, Comm. Math. Phys., 76 (1980), 65-116.

[28] Clarkson, P. A., Gordoa, P. R. and Pickering, A., Multicomponent equations associated to non-isospectral scattering problems, Inverse Problems, 13 (1997), 1463-1476.

[29] Jimbo, M., Miwa, T. and Ueno, K., Monodromy preserving deformations of linear ordinary differential equations with rational coefficients, I, Physica D, 2 (1981), 306-352.

[30] Jimbo, M. and Miwa, T., Monodromy preserving deformations of linear ordinary differential equations with rational coefficients, II, Physica D, 2 (1981), 407-448. 
[31] Jimbo, M. and Miwa, T., Monodromy preserving deformations of linear ordinary differential equations with rational coefficients, III, Physica D, 4 (1981), 26-46.

[32] Clarkson, P. A., Joshi, N. and Pickering, A., Bäcklund transformations for the second Painlevé hierarchy: a modified truncation approach, Inverse Problems, 15 (1999), 175187. 